Original Research Paper

\title{
Effect of Serial Passaging on the Morphology and Biological Characteristics of Human Adipose Tissue-derived Stem Cells
}

\author{
${ }^{1}$ Lenka Oravcova, ${ }^{2}$ Martin Bohac, ${ }^{3}$ Lubica Krajciova, \\ ${ }^{1}$ Stefan Polak, ${ }^{3}$ Daniel Bohmer and ${ }^{3}$ Lubos Danisovic \\ ${ }^{1}$ Institute of Histology and Embryology, Faculty of Medicine, Comenius University, Bratislava, Slovakia \\ ${ }^{2}$ Department of Plastic Surgery, Faculty of Medicine, Comenius University, Bratislava, Slovakia \\ ${ }^{3}$ Institute of Medical Biology, \\ Genetics and Clinical Genetics, Faculty of Medicine, Comenius University, Bratislava, Slovakia
}

Article history

Received: 03-06-2016

Revised: $24-10-2016$

Accepted: 14-12-2016

Corresponding Author:

Lubos Danisovic

Institute of Medical Biology,

Genetics and Clinical Genetics,

Faculty of Medicine, Comenius

University, Bratislava, Slovakia

Email: lubos.danisovic@fmed.uniba.sk

\begin{abstract}
Somatic stem cells posses an exclusive property of self regeneration and plasticity which makes them promising candidates for use in tissue engineering and regenerative medicine. In the case of stem cell therapy it is crucial to obtain them in sufficient amount. That's why they have to be cultured under in vitro conditions. Long term culture provides adequate number of cells, but on the other hand it is associated with increased risk of spontaneous cell transformation. In this study we analyzed the effect of serial passaging (up to 10th passage) on the morphological and selected biological characteristics of human stem cells isolated from adipose tissue in respect to potential malignant transformation. Obtained results showed that human adipose tissue-derived stem cells maintained their morphological and biological properties during 10 passages and after performing further tests mainly addressed the cytogenetic stability and expression of regulators of cell cycle, senescence and apoptosis, they should be safely used in clinical practice.
\end{abstract}

Keywords: Stem Cells, Adipose Tissue, Long Term Culture, Morphology, Biological Properties

\section{Introduction}

Mesenchymal Stem Cells (MSCs) are defined as clonal and multipotent cells that can undergo the process of multilineage differentiation. They also posses unique capability of self-renewing and active migration to affected tissues or organs. In mature organism they are responsible for promoting the process of regeneration of damaged tissues (Shanti et al., 2007). Due to these biological properties they attracted significant attention mainly in field of regenerative medicine and tissue engineering (Richardson et al., 2016; Zamborsky et al., 2014).

MSCs were first obtained from bone marrow as fibroblastic Colony Forming Units (CFU-F) by Friedenstein et al. (1976). Since their discovery, MSCs were isolated from different tissues, including cord blood, placenta, skin, skeletal muscles, periosteum, etc. MSCs are characterized by specific markers-CD73, CD90 and CD105; and do not produce markers of hematopoietic and endothelial cells-CD14, CD34 and CD45. Moreover, they must be capable of differentiating into at least three lineages (Dominici et al., 2006). Recent research focused on genomic profiles provided evidence that MSCs express also markers of pluripotency (Oct-4, Nanog and Sox2), which maintained their undifferentiated state (De Los Angeles et al., 2015). Moreover, Kestendjieva et al. (2008) showed that MSCs produce survivin, which is also extensively expressed in majority of human cancers (Adamkov et al., 2015; Halasova et al., 2010).

Recently, adipose tissue belong to most easily accessible source of MSCs. Sampling procedure (liposuction) is relative safe and does not represent increased risk for patient when compared with other tissue sources. Moreover, $1 \mathrm{~g}$ of adipose tissue contains approximately 500-5000 of MSCs, which may be obtained by enzymatic digestion with subsequent cultivation in vitro (Sakaguchi et al., 2005).

The basic prerequisite for clinical application of MSCs is their therapeutically sufficient quantity. However, it was reported that prolonged cultivation may affect properties of MSCs and may give arise to spontaneous transformation (Kuniakova et al., 2015). 
In the present study we analyzed morphological and selected biological characteristics of MSCs obtained from adipose tissue during pro-longed expansion in vitro (up to 10th passage).

\section{Materials and Methods}

\section{Isolation and Cell Culture}

Adipose Tissue-Derived Stem Cells (ATSCs) were obtianed from fresh subcutaneous fat tissue obtained during planed aesthetic surgeries. All donors were healthy females $(n=6$; average age $=38$ years $)$ and all procurement procedures were performed in agreement with Helsinki declaration after obtaining patient's informed consent.

ATSCs were isolated according protocol published by Zuk et al. (2001) with several modifications. Briefly, lipoaspirates were washed with Phosphate Buffered Saline (PBS; Oxoid, GB) supplemented with $100 \mu \mathrm{g}$ $\mathrm{mL}^{-1}$ streptomycin, $100 \mathrm{U} \mathrm{mL}^{-1}$ penicillin and $0.25 \mu \mathrm{g}$ $\mathrm{mL}^{-1}$ Amphotericine B (PAA, Austria). Aliquots of $8 \mathrm{~g}$ were taken into $50 \mathrm{~mL}$ centrifuge tubes with $0.1 \%$ collagenase type I (Gibco, USA) and incubated for 60 min at $37^{\circ} \mathrm{C}$ on a shaker. Subsequently, samples were centrifuged (10 min, $1200 \mathrm{rpm}$ ). The supernatants were aspired. Obtained sediments were re-suspended with complete culture medium consisted of DMEM-Ham's F12 (Sigma-Aldrich, USA) supplemented with $10 \%$ fetal bovine serum (FBS; PAA, Austria) and $100 \mu \mathrm{g} \mathrm{mL}^{-1}$ streptomycin, $100 \mathrm{U} \mathrm{mL} \mathrm{mL}^{-1}$ penicilin, $0.25 \mu \mathrm{gL}^{-1}$ Amphotericine B followed by next centrifugation. Final pellets were re-suspended with complete culture medium and filtered through $40 \mu \mathrm{m}$ cell strainers into culture flasks (TPP, Switzerland). ATSCs were maintained under standard conditions in $\mathrm{CO}_{2}$ incubator $\left(37^{\circ} \mathrm{C} ; 5 \%\right.$ $\mathrm{CO}_{2}$ ) for $24 \mathrm{~h}$. Then, non-adherent cells were aspirated by changing the medium. Culture medium was refreshed every 2-3 days. When the cells reached $80 \%$ confluence, they were trypsinized by $0.25 \%$ trypsine (Sigma-Aldrich, USA) and sub-cultured at 5000 cells $/ \mathrm{cm}^{2}$ under same condition until 10th passage.

\section{Immunophenotyping}

ATSCs appointed for flow cytometric analysis were detached by $0.25 \%$ trypsin and then re-suspended in blocking buffer (solution of PBS and $0.5 \%$ bovine serum albumin) (PAA, Austria). The MSC phenotyping kit (Miltenyi Biotec, Germany) was applied in all experiments. Cell suspensions were processed according to manufacturer's protocol and were analyzed by MACSQuant ${ }^{\circledR}$ Analyzer (Miltenyi Biotec, Germany).

\section{Morphological Analysis}

The morphology of ATSCs was gradually evaluated using inverted microscope Zeiss Axiovert 100 (Carl Zeiss, Germany).
ATSCs selected for TEM analysis were fixed in $2.5 \%$ glutaraldehyde (Sigma-Aldrich, USA), $\mathrm{pH}=7.2$, at $4{ }^{\circ} \mathrm{C}$ for $4 \mathrm{~h}$. Subsequently, cells were washed by PBS and post-fixed using $2 \%$ osmium tetraoxide (Serva, Germany) for $2 \mathrm{~h}$, then washed in distilled water and dehydrated in a graduated series of ethanol (Centralchem, Slovakia). Afterwards, the samples were embedded in Durcupan (Sigma-Aldrich, USA) and sliced into semi-thin sections. The samples were stained by toluidine blue (Sigma-Aldrich, USA) for $10 \mathrm{~min}$ and minced into ultrathin sections. Finally, they were mounted on 200 mesh copper grids, double stained by uranyl acetate and lead citrate (Sigma-Aldrich, USA) and analyzed using a TEM FEI Morgagni 268D (FEI, USA).

\section{Cell Cycle Analysis}

ATSCs were detached with $0.25 \%$ trypsine and centrifuged (1200 rpm, $10 \mathrm{~min})$. Obtained cells were carefully rinsed with PBS followed by ethanol fixation. Prior to analysis, cells were treated with Muse Cell Cycle Reagent (Merck Millipore, USA) for $30 \mathrm{~min}$. Cells were assessed with Muse Cell Analyzer (Merck Millipore, USA).

\section{Detection of Telomerase Activity}

Detection of telomerase activity of ATSCs was continually analyzed by using TRAPeze ${ }^{\circledR}$ RT Telomerase Detection Kit (Merck Millipore, USA) according to the manufacturer's recommendations. Briefly, the cells were resuspended in $200 \mu \mathrm{L}$ CHAPS Lysis Buffer (Merck Millipore, USA) and incubated on ice for $30 \mathrm{~min}$. Then, cells were processed by centrifugation $(12000 \mathrm{rpm} ; 20 \mathrm{~min}$ at $\left.4^{\circ} \mathrm{C}\right) .160 \mu \mathrm{L}$ of the supernatant was transferred into a tube and the protein concentration was determined. Finally, the "Master Mix" was performed by mixing all reagents with sample. The PCR reactions were done on Eco RealTime PCR System (Illumina, USA).

\section{Cytogeneic Analysis}

Karyological slides were prepared according to standard protocol. Briefly, ATSCs appointed for cytogenetic analysis were treated by colcemid solution. After $2 \mathrm{~h}$, cells were trypsinized. Obtained suspension was resuspended in culture medium and centrifuged. Supernatant was aspirated and cells were hypotonized by $0.075 \mathrm{M} \mathrm{KCl}$. After subsequent centrifugation, cells were two times fixed by pre-cooled fixation solution (methanol and acetic acid). Cells were pipetted on the clean slide and stained by Giemasa solution (Centralchem, Slovakia).

\section{Results}

ATSCs were attached on the bottom of culture flasks after $24 \mathrm{~h}$ of culture. They proliferated in colonies and had typical fibroblast-like morphology (Fig. 1a). Between 3 to 5 days, ATSCs reached $80 \%$ confluence 
and were sub-passaged. During further cultivation (up to 10th passage) they keep this morphology (Fig. 1a-d).

The results (Fig. 2) from cytometric analysis proved the expression surface antigens CD73, CD90 and CD105 typical for MSCs. However, the expression of CD73 in 10th passage was not that homogenic compared to expression recorded in previous passages. On the other hand, they did not express CD14, CD20, CD34 and CD45. The expression of these surface antigens was not altered even when ATSCs were cultured up to passage 10 .
As presented on Fig. 3, morphological analysis on TEM level showed normal ultrastructural feature of protheosynthetically active cells. They had irregular morphology with noticeable nuclei (often eccentrically located). They contain predominantly euchromatine. ATSCs contained well developed rough endoplasmic reticulum (Fig. 3a-b). The first changes in ultrastructural morphology were recorded 10th passage. ATSCs contained lamellar bodies, so called endosomes in their cytoplasm (Fig. 3c).
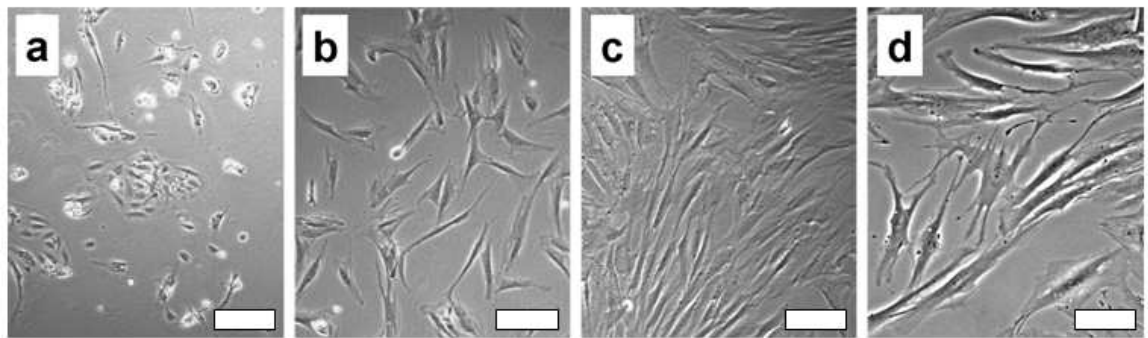

Fig. 1. Representative micrographs of in vitro expanded human ATSCs: (a) primary culture; (b) 1st passage; (c) 5th passage; (d) 10th passage $($ scale bar $=20 \mu \mathrm{m})$

P0

P1

P3

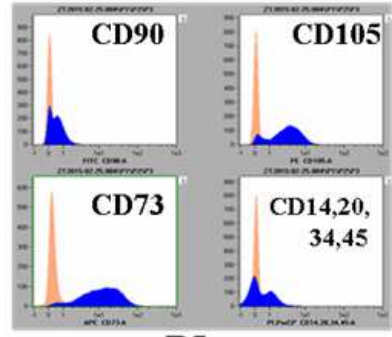

P5
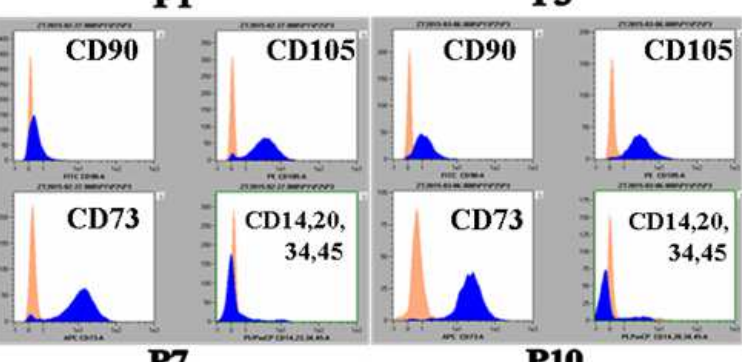

P7
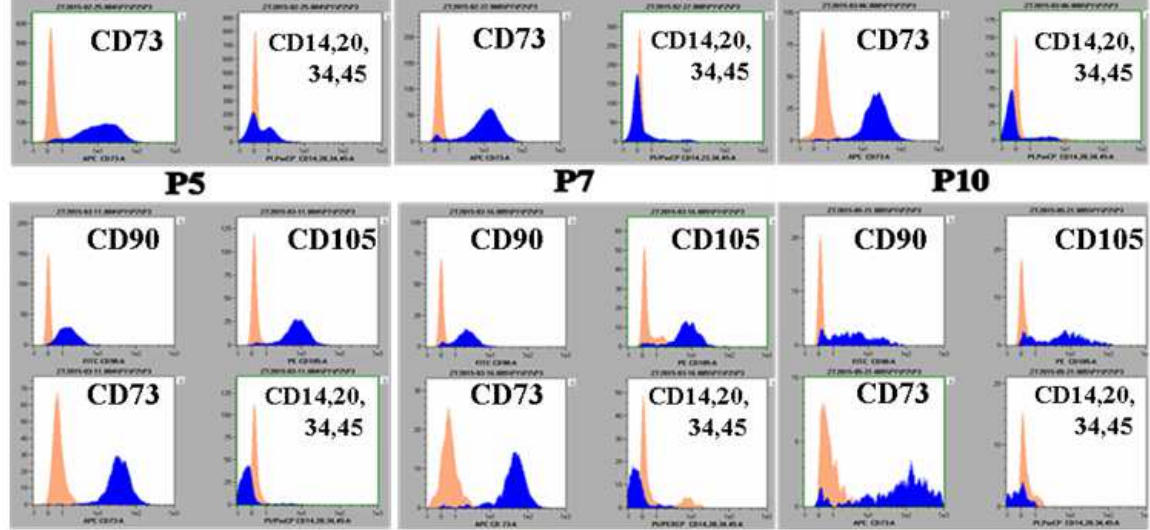

Fig. 2. Representative histograms of human ATSCs during prolonged in vitro expansion

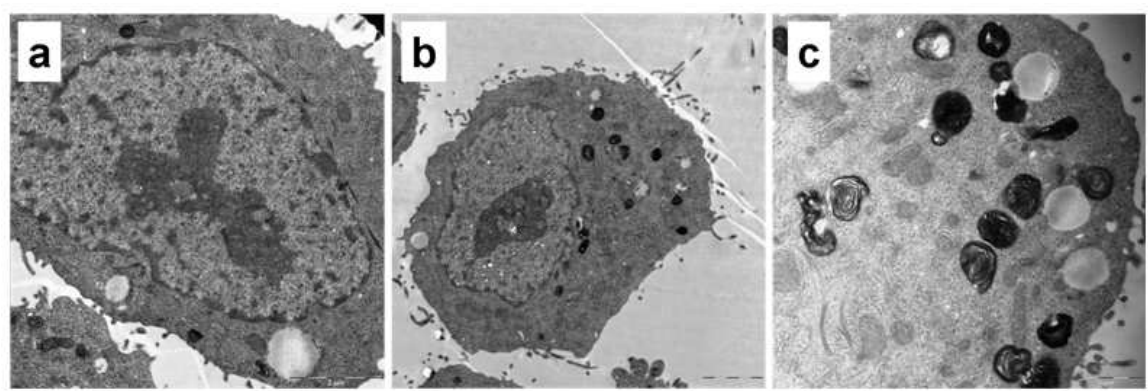

Fig. 3. Representative electronograms of in vitro expanded human ATSCs: (a) 1st passage; (b) 5th passage; (c) 10th passage 
P1

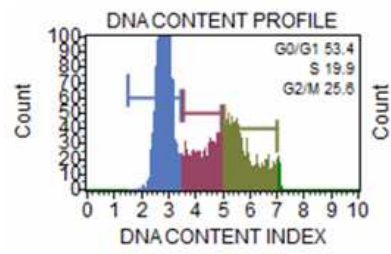

P5

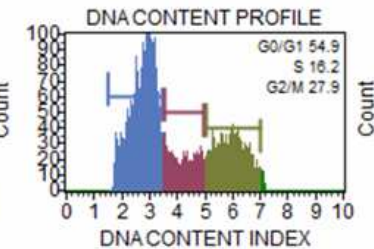

P10

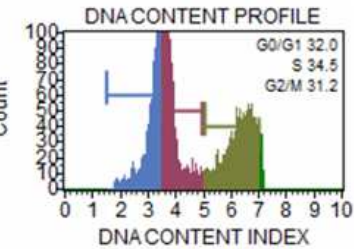

Fig. 4. The representative distribution of certain cell cycle phases during prolonged in vitro expansion of human ATSCs

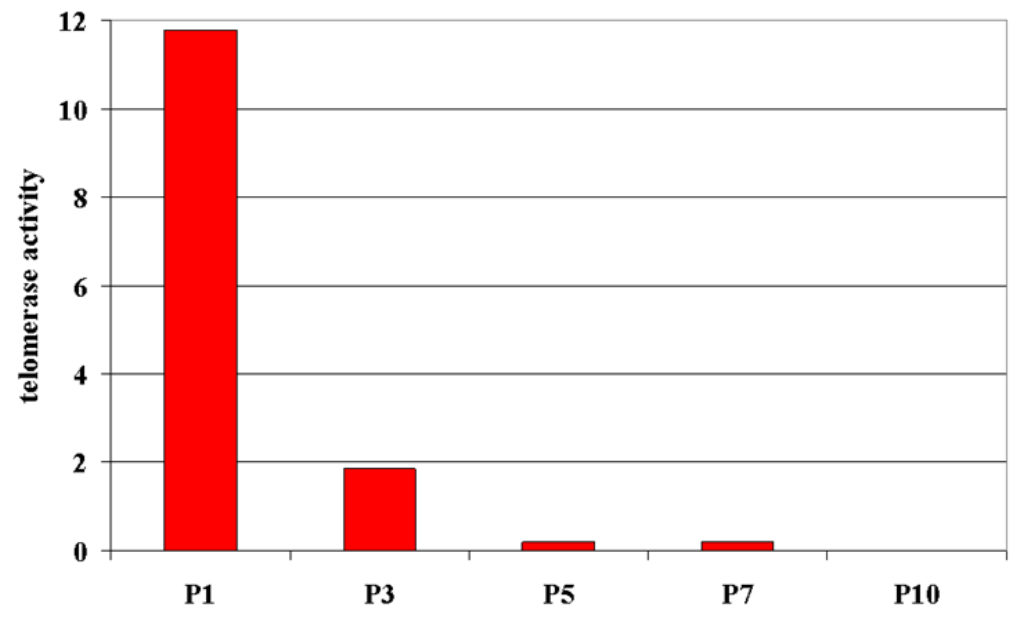

Fig. 5. Telomerase activity in human ATSCs during prolonged in vitro expansion
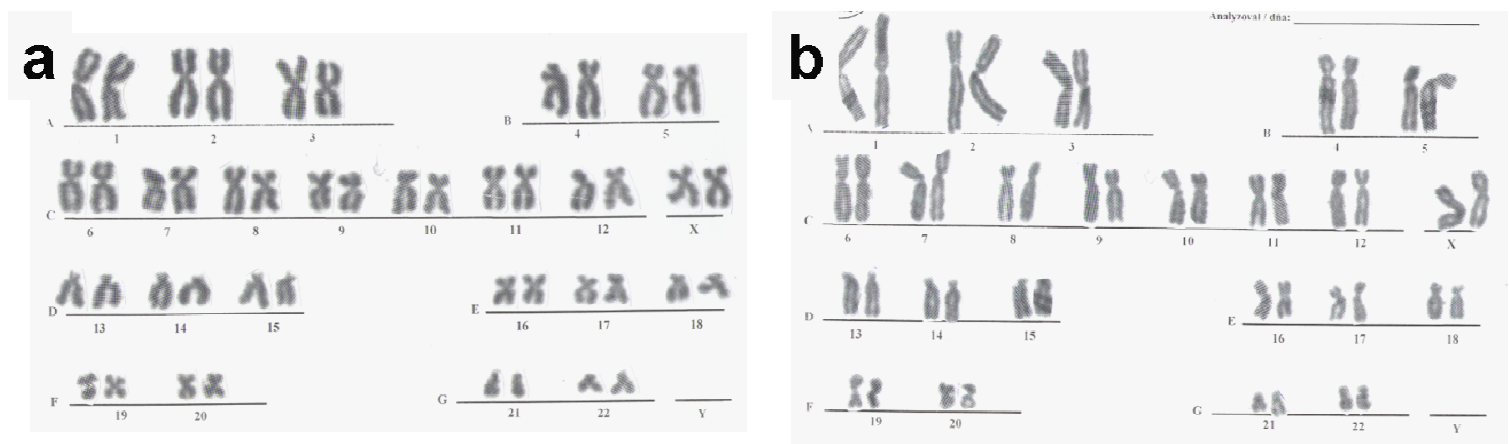

Fig. 6. Representative karyotypes of human ATSCs during prolonged in vitro expansion: (a) 1st passage; (b) 10th passage

The distribution of certain cell cycle phases is presented in Fig. 4 and Table 1. During early passages (P1-P5), the majority of cells were arrested in G0/G1 phase. In later passages ATSCs were characterized by increased proliferation, majority of cells were in $\mathrm{S}$ and G2/M phase.

As presented in Fig. 5, the TRAPeze assay demonstrated that hADSCs gradually lost telomerase activity in passage increasing manner.

Cytogenetic analysis did not show any changes in karyotype. During whole experiment (up to 10th passage) the ATSCs had normal female karyotype 46,XX (Fig. 6).

\section{Discussion}

Somatic stem cells which were first described over fifty years ago still attract tremendous interest of the researchers nowadays. It was documented, that stem cells due to their unique biological characteristics have significant therapeutic potential for repairing damaged and/or defective tissues (Ham et al., 2015; Sekiya et al., 2015; Zamborsky et al., 2014). The main prerequisite of stem cell-based therapies application is obtaining their adequate amount by expansion in vitro. The time of culture seem to be critical because several studies described changes of biological properties of stem cells 
during prolonged cultivation (Nava et al., 2015; Zhu et al., 2015). The present study was therefore aimed at analysis of selected biological and morphological properties of ATSCs during prolonged culture in vitro up to 10th passage.

ATSCs were obtained from fresh subcutaneous fat of healthy donors by enzymatic digestion. This approach has been used to obtain population of fibroblast-like stem cells in high quantities, up to $1.10^{6} / \mathrm{g}$ of adipose tissue (Yang et al., 2011). Due to the yield and the fact that liposuction represent only low burden to patients, adipose tissue represent attractive, safe and rich source of adult stem cells. After isolation, ATSCs start to proliferate in colonies and display high proliferation potential. ATSCs were characterized by flow cytometry. They expressed markers of mesenchymal stem cells CD73, CD90 and CD105; and did not produce CD14, CD20, CD34 and CD45 typical for haematopoietic and endothelial cells (Dominici et al., 2006). During all experiment, we did not record alterations in the production of mentioned markers, instead of nonhomogenic expression of CD73 in 10th passage. Mentioned may be associated with different intensity of CD73 expression in cultured ATSCs (cells became more heterogenous). This phenomenon may be overlapped by selecting more suitable culture media. However, our results correlated with results of Shi et al. (2015) who showed stable immunophenotype of human umbilical cord-derived MSCs up to passage 16.

Morphological analysis showed the morphology of fibroblast-like cells. On the level of light microscope, we observed only small alternation of morphology in the passage 10; cells became bigger in their cytoplasmic projections. Mentioned was in good correlation with finding obtained from TEM analysis. At the ultrastructural level, ATSCs showed morphology of proteosynthetically active cells without pathological changes. Cells contained nuclei with prevalence of euchromatine. They were also characterized by well developed rough endoplasmic reticulum. Only in last passage they contained a small amount of lamellar bodies, s.c. endosomes in the cytoplasm. In our previous morphological study we identify these structures in higher passages (about passage 30) but they were present in significantly higher quantities (they filled out almost whole cytoplasm) (Varga et al., 2015). These structures represent late stage of endosome maturation with partial electron dense areas prior to final breakdown by lysosomes (Huotari and Helenius, 2011).

The analysis of cell cycle showed that ATSCs were arrested in G0/G1 phase at the beginning of experiment. Mentioned is probably associated with process of adaptation on in vitro conditions. In later passages, ATSCs underwent massive proliferation and majority of them were in $\mathrm{S}$ and $\mathrm{G} 2 / \mathrm{M}$ phase. Comparable finfings were described by other authors dealing with different stem cells (Chen et al., 2014; Jiang et al., 2012).
Our data obtained from analysis of telomerase activity showed, that serial passaging led in its reduction in passage increasing manner. This was in agreement with previous studies dealing with stem cells from different sources in prolonged in vitro culture (Nava et al., 2015; Fu et al., 2015). However, there is still possibility of alternative lengthening of telomeres (maintained by homologous recombination-mediated DNA replication mechanism) which occurs in some human tumours of mesenchymal origin (Cesare and Reddel, 2010). Nava et al. (2015) analyzed ALT in hADSCs during long term culture, but their results did not proved its presence.

Another important finding was that ATSCs during the first 10 passages maintained the cytogenetic stability. However, it should be noted that we have used only the conventional staining and therefore it will be useful in the future to add additional cytogenetic analysis (e.g., Comet assay for DNA fragmentation, micronucleus test etc.) because other authors captured in high passages not only of numerical aberrations but also translocation, chromosomal breaks etc. (Froelich et al., 2013).

\section{Conclusion}

Based on obtained results we can emphasize that in vitro cultured human ATSCs during the first 10 passages do not exhibit substantial changes in morphology and in biological properties. However, in the context of their safe use in clinical practice we recommend to perform further tests mainly addressed the cytogenetic stability and expression of regulators of cell cycle, senescence and apoptosis.

\section{Acknowledgement}

The authors thank Mrs. Haraslinova for processing biological material for TEM analysis.

\section{Funding Information}

This work was supported by grants of Ministry of Health of the Slovak Republic No. 2012/4-UKBA-4, Slovak Research and Development Agency No. 0434-12 and Comienius University No. 109/2016.

\section{Author's Contributions}

Lenka Oravcova: Participates in all experiments, review literature and write manuscript.

Lubica Krajciova: Participates in analysis of telomerase activity and cytogenetic evaluation.

Martin Bohac: Perform sampling procedures during surgeries.

Stefan Polak: Perform morphological analysis.

Daniel Bohmer: Participates in cytogenetic evaluation and contributed to the writing manuscript.

Lubos Danisovic: Designed the research plan, organized the study and contributed to result analysis and the writing manuscript. 


\section{Ethics}

This article is original and contains unpublished material. The corresponding author confirms that all of the other authors have read and approved the manuscript and no ethical issues involved.

\section{References}

Adamkov, M., D. Vybohova, V. Tupa, J. Chylíková and J. Horáček et al., 2015. Expression and significance of survivin in colorectal high grade and low grade adenomas. Acta Histochem., 117: 590-594. DOI: $10.1016 /$ j.acthis.2015.05.006

Cesare, A.J. and R.R. Reddel, 2010. Alternative lengthening of telomeres: Models, mechanisms and implications. Nat. Rev. Genet., 11: 319-330. DOI: $10.1038 / \mathrm{nrg} 2763$

Chen, G., A. Yue and Z. Ruan, Y. Yin and R. Wang et al., 2014. Monitoring the biology stability of human umbilical cord-derived mesenchymal stem cells during long-term culture in serum-free medium. Cell Tissue Bank., 15: 513-521.

DOI: $10.1007 / \mathrm{s} 10561-014-9420-6$

De Los Angeles, A., F. Ferrari and R. Xi, R. Xi and Y. Fujiwara et al., 2015. Hallmarks of pluripotency. Nature, 525: 469-478. DOI: 10.1038/nature15515

Dominici, M., K. LeBlanc, I. Mueller, I. SlaperCortenbach and F.C. Marini et al., 2006. Minimal criteria for defining multipotent mesenchymal stromal cells. The International Society for Cellular Therapy position statement. Cytotherapy, 8: 315-317. DOI: $10.1080 / 14653240600855905$

Friedenstein, A.J., J.F. Gorskaja and N.N. Kulagina, 1976. Fibroblast precursors in normal and irradiated mouse hematopoietic organs. Exp. Hematol., 4: 267-274. PMID: 976387

Froelich, K., J. Mickler, G. Steusloff, A. Technau and M.R. Tirado et al., 2013. Chromosomal aberrations and deoxyribonucleic acid single-strand breaks in adipose-derived stem cells during long-term expansion in vitro. Cytotherapy, 15: 767-781.

DOI: 10.1016/j.jcyt.2012.12.009

Fu, W.L., J. Li, G. Chen, T. Xin and Z. Cheng-Hao et al., 2015. Mesenchymal stem cells derived from peripheral blood retain their pluripotency, but undergo senescence during long-term culture. Tissue Eng. Part C Methods., 21: 1088-1097.

DOI: $10.1089 /$ ten.TEC.2014.0595

Halasova, E., M. Adamkov, T. Matakova, E. Kavcova and I. Poliacek et al., 2010. Lung cancer incidence and survival in chromium exposed individuals with respect to expression of anti-apoptotic protein survivin and tumor suppressor P53 protein. Eur. J. Med. Res., 15: 55-59.

DOI: $10.1186 / 2047-783 \mathrm{X}-15-\mathrm{S} 2-55$
Ham, O., C.Y. Lee, R. Kim, J. Lee and S. Oh et al., 2015. Therapeutic potential of differentiated mesenchymal stem cells for treatment of osteoarthritis. Int. J. Mol. Sci., 16: 14961-14978. DOI: $10.3390 /$ ijms 160714961

Huotari, J. and A. Helenius, 2011. Endosome maturation. EMBO J., 30: 3481-3500. DOI: $10.1038 / \mathrm{emboj} .2011 .286$

Jiang, L., T. Liu and K. Song, 2012. Growth characteristics of human adipose-derived stem cells during long time culture regulated by cyclin a and cyclin D1. Applied Biochem. Biotechnol., 168: 2230-2244. DOI: $10.1007 / \mathrm{s} 12010-012-9932-0$

Kestendjieva, S., D. Kyurkchiev and G. Tsvetkova, 2008. Characterization of mesenchymal stem cells isolated from the human umbilical cord. Cell Biol. Int., 32: 724-732. DOI: 10.1016/j.cellbi.2008.02.002

Kuniakova, M., L. Oravcova, Z. Varchulova-Novakova, D. Viglaska and L. Danisovic et al., 2015. Somatic stem cell aging and malignant transformation impact on therapeutic application. Cell. Mol. Biol. Lett., 20: 743-756. DOI: 10.1515/cmble-2015-0045

Nava, M.B., G. Catanuto, A.E. Pennati, A.E. Pennati and N. Rocco et al., 2015. Lack of activation of telomere maintenance mechanisms in human adipose stromal cells derived from fatty portion of lipoaspirates. Plast. Reconstr. Surg., 135: 114e-123e. DOI: $10.1097 /$ PRS.0000000000001008

Richardson, S.M., G. Kalamegam, P.N. Pushparaj, C. Matta and A. Memic et al., 2016. Mesenchymal stem cells in regenerative medicine: Focus on articular cartilage and intervertebral disc regeneration. Methods, 99: 69-80.

DOI: 10.1016/j.ymeth.2015.09.015

Sakaguchi, Y., I. Sekiya, K. Yagishita and T. Muneta, 2005. Comparison of human stem cells derived from various mesenchymal tissues: Superiority of synovium as a cell source. Arthritis Rheum., 52: 2521-2529. DOI: 10.1002/art.21212

Sekiya, I., T. Muneta, M. Horie and H. Koga, 2015. Arthroscopic transplantation of synovial stem cells improves clinical outcomes in knees with cartilage defects. Clin. Orthop. Relat. Res., 473: 2316-2326. DOI: $10.1007 / \mathrm{s} 11999-015-4324-8$

Shanti, R.M., W.J. Li, L.J. Nesti, X. Wang and R.S. Tuan et al., 2007. Adult mesenchymal stem cells: Biological properties, characteristics and applications in maxillofacial surgery. J. Oral. Maxillofac Surg., 65: 1640-1647. DOI: $10.1016 /$ j.joms.2007.04.008

Shi, Z., L. Zhao, G. Qiu, X.J. Su and Z.Y. Dong et al., 2015. High efficient isolation and systematic identification of human adipose-derived mesenchymal stem cells. Mol. Cell Biochem., 401: 155-164. DOI: 10.1186/1423-0127-18-59 
Varga, I., M. Miko, L. Oravcova, T. Bačkayová and J. Koller et al., 2015. Ultra-structural morphology of long-term cultivated white adipose tissue-derived stem cells. Cell Tissue Bank., 16: 639-647. DOI: $10.1007 / \mathrm{s} 10561-015-9513-\mathrm{X}$

Yang, X.F., X. He, J. He, L.H. Zhang and X.J. Su et al., 2011. High efficient isolation and systematic identification of human adipose-derived mesenchymal stem cells. J. Biomed. Sci., 18: 59-59. DOI: $10.1186 / 1423-0127-18-59$

Zamborsky, R., J. Rusnakova and A. Nicodemou, 2014. Tissue engineering of articular cartilage: A minireview. OnLine J. Biol. Sci., 14: 248-253.

DOI: $10.3844 /$ ojbsci.2014.248.253
Zhu, Y., X. Song, J. Wang, Y. Li and Y. Yang et al., 2015. Placental mesenchymal stem cells of fetal origin deposit epigenetic alterations during longterm culture under serum-free condition. Expert Opin. Biol. Ther., 15: 163-180. DOI: $10.1517 / 14712598.2015 .960837$

Zuk, P.A., M. Zhu, H. Mizuno, J.W. Futrell and A.J. Katz et al., 2001. Multilineage cells from human adipose tissue: Implications for cell-based therapies. Tissue Eng., 7: 211-228.

DOI: $10.1089 / 107632701300062859$ 